\title{
Farmers' satisfaction with agricultural extension service quality in Ninh Phuoc district, Ninh Thuan province
}

\author{
Hoang M. Do*, \& Nam H. Tran
}

Faculty of Economics, Nong Lam University, Ho Chi Minh City, Vietnam

ARTICLE INFO
Research paper
Received: October 27, 2017
Revised: January 31, 2018
Accepted: February 05, 2018
Keywords
Agricultural extension program
Households
Satisfaction
*Corresponding author
Do Minh Hoang
Email: dominhhoang@hcmuaf.edu.vn

\begin{abstract}
Agricultural extension service quality is a key to sustainable development and enhances the efficiency of government resources for farmers. This paper assessed farmer's satisfaction with the quality of agricultural extension services in Ninh Phuoc district, Ninh Thuan province. Major analytical tools included: exploratory factor analysis (EFA), confirmatory factor analysis (CFA) and structural equation modeling (SEM). The data were collected by direct interviews with 320 farmers participating on the extension. The analysis results showed that the level of satisfaction of the farmers was significantly influenced by the quality of the agricultural extension service and the agricultural extension service quality was influenced by factors such as farmers's adoption of technological innovations in agriculture, the extension staff's clear presentation and understandable instructions to farmers, experienced trainers, exciting and pleasant discussions and field trip activities tailored to the needs of farmers.
\end{abstract}

Cited as: Do, H. M., \& Tran, N. H. (2018). Farmers' satisfaction with agricultural extension service quality in Ninh Phuoc district, Ninh Thuan province. The Journal of Agriculture and Development $17(4), 1-10$. 


\title{
Đánh giá mức độ hài lòng của nông hộ đối với chất lượng dịch vụ chương trình khuyến nông tại huyện Ninh Phước, tỉnh Ninh Thuận
}

\author{
Đỗ Minh Hoàng* \& Trần Hoài Nam
}

Khoa Kinh Tế, Trường Đại Học Nông Lâm TP. Hồ Chí Minh, TP. Hồ Chí Minh

\section{THÔNG TIN BÀI BÁO}

\section{Bài báo khoa học}

Ngày nhận: 27/10/2017

Ngày chỉnh sửa: $31 / 01 / 2018$

Ngày chấp nhận: 05/02/2018

\section{Từ khóa}

Chương trình khuyến nông

Mức độ hài lòng

Nông hộ

*Tác giả liên hệ

Đỗ Minh Hoàng

Email: dominhhoang@hcmuaf.edu.vn

\section{TÓM TẮT}

Chất lượng dịch vụ chương trình khuyến nông là chìa khóa cho vấn đề phát triển bền vững và nâng cao hiệu quả những nguồn lực của Chính phủ dành cho nông dân. Bài viết này đánh giá mức độ hài lòng của nông hộ đối với chất lượng dịch vụ chương trình khuyến nông tại huyện Ninh Phước, tỉnh Ninh Thuận. Các công cư phân tích chính bao gồm: phân tích nhân tố khám phá (EFA), phân tích nhân tố khẳng định (CFA) và mô hình cấu trúc tuyến tính (SEM). Số liệu được thu thập bằng cách phỏng vấn trực tiếp 320 nông hộ tham gia khuyến nông trên địa bàn. Kết quả phân tích cho thấy mức độ hài lòng của nông hộ chịu ảnh hưởng khá lớn từ chất lượng dịch vụ chương trình khuyến nông và chất lượng dịch vụ chương trình khuyến nông bị ảnh hưởng bởi các nhân tố như nông hộ an tâm khi áp dụng các tiến bộ trong sản xuất; cán bộ khuyến nông trình bày rõ ràng, hướng dẫn dễ hiểu, có nhiều kinh nghiệm; trao đổi trong lớp học sôi nổi, thoải mái và hoạt động tham quan thực tế phù hợp với nhu cầu của người nông dân.

\section{1. Đắt Vấn Đề}

Khuyến nông đã được hình thành và phát triển khá lâu trên thế giới, mỗi giai đoạn phát triển của nền nông nghiệp lại có một mô hình khuyến nông đặc trưng. Ở các nước đang phát triển, nền nông nghiệp chưa hiện đại, mô hình khuyến nông chủ yếu dựa vào sự tham gia của nhiều bên và mô hình khuyến nông đặc trưng cho sự vận hành cả hệ thống khuyến nông của mỗi quốc gia (Nguyen \& Nguyen, 2016). Tại Việt Nam, hệ thống khuyến nông đã trở thành một công cụ hữu hiệu và là cầu nối quan trọng trong chuyển giao tiến bộ kỹ thuật, công nghệ mới, đóng góp vào sự tăng trưởng thành công của sản xuất nông nghiệp. Mặt khác, để hệ thống khuyến nông hoạt động hiệu quả góp phần duy trì tốc độ tăng trưởng của ngành nông nghiệp thì 3 yếu tố có tính quyết định lớn là chủ thể tổ chức các hoạt động khuyến nông, loại hình các hoạt động khuyến nông và kinh phí cho khuyến nông.

Trong thời gian qua, ngành nông nghiệp huyện Ninh Phước đã và đang triển khai nhiều chương trình khuyến nông nhằm chuyển giao các tiến bộ khoa học kỹ thuật trong canh tác nông nghiệp cho nông dân.Tuy nhiên, trước những thách thức mới cho một nền nông nghiệp phát triển bền vững, công tác này vẫn bộc lộ những hạn chế nhất định, việc đúc kết chất lượng các chương trình khuyến nông vẫn còn bỏ ngõ và để làm được điều này cần phải thấu hiểu được mức độ hài lòng của nông dân đối với chất lượng dịch vụ chương trình khuyến nông. Kết quả nghiên cứu là căn cứ khoa học cho ngành Nông nghiệp huyện Ninh Phước, tỉnh Ninh Thuận tham khảo để xây dựng chương trình mang tính thiết thực và hữu ích hơn trong thời gian tới.

\section{Cơ Sở Lý Luận và Phương Pháp Nghiên Cứu}

\subsection{Tổng quan tài liệu}

Chất lượng là tất cả đặc điểm, đặc tính của sản phẩm, dịch vụ liên quan tới khả năng làm thỏa mãn những nhu cầu hàm ẩn hoặc được xác định. Sản phẩm hoặc dịch vụ có chất lượng khi nó đáp ứng hoặc vượt mong đợi của khách hàng (Kotler 
\& Keller, 2006). Hài lòng là cảm giác vui thích hoặc thất vọng bắt nguồn từ sự so sánh thể hiện của sản phẩm dịch vụ cảm nhận được với mong đợi của khách hàng (Lin, 2003; Kotler \& Keller, 2006). Quan hệ giữa chất lượng dịch vụ và sự hài lòng (Parasuraman \& ctv., 1988) cho rằng chất lượng dịch vụ và hài lòng có liên quan nhau, trong đó, hài lòng qua thời gian có từ cảm nhận về chất lượng dịch vụ. Cronin \& Taylor (1992) đưa ra kêt quả nghiên cứu khuyến cáo là chất lượng dịch vụ là tiền tố của sự hài lòng của khách hàng. Theo Olajide (2011), chất lượng dịch vụ và sự hài lòng có liên quan nhau. Do đó, không nên đo lường chất lượng dịch vụ mà không đánh giá sự hài lòng của khách hàng.

Elias \& ctv. (2015) đã nghiên cứu sự hài lòng của người nông dân đối với chương trình khuyến nông và các yếu tố ảnh hưởng tại miền Tây Bắc Ethiopia. Bằng việc sử dụng mô hình hồi quy Logit thứ tự, nghiên cứu cho thấy lợi nhuận kinh tế, sự liên hệ với khuyến nông thường xuyên, quy mô hộ gia đình và thu nhập phi nông nghiệp tác động mạnh đến sự hài lòng của nông hộ; mặt khác, sự giới hạn về công nghệ, giá cả yếu tố đầu vào cao, hệ thống cho vay không thuận tiện là một trong những nguyên nhân được cho là làm giảm sự hài lòng của nông dân.

Wayne \& ctv. (2014) đã nghiên cứu sự hài lòng của nông dân đối với chương trình khuyến nông tại các bang miền đông Caribe. Nghiên cứu này sử dụng thang đo Likert và mô hình hồi quy đa biến để xác định các nhân tố ảnh hưởng đến sự hài lòng của nông dân như giới tính của chủ hộ, quy mô đất, trình độ học vấn, số lần tham quan trình diễn, cách tiếp cận nguồn thông tin khuyến nông.

Pham \& ctv. (2014) đã phân tích các yếu tố ảnh hưởng sự hài lòng của nông dân qua khoá học tập huấn FFS về tăng cường kỹ năng chọn giống và sản xuất lúa giống cộng đồng tỉnh Hậu Giang năm 2012. Kết quả nghiên cứu cho thấy, sự hài lòng của nông dân chịu ảnh hưởng của các yếu tố như: Độ tin cậy của lớp tập huấn; cơ sở vật chất và điều kiện học tập; khả năng đáp ứng yêu cầu của lớp học; sự đảm bảo của lớp học; sự cảm thông của giảng viên.

Nguyen \& ctv. (2011) đã phân tích mức độ hài lòng của nông hộ đối với phương pháp tập huấn ứng dụng tiến bộ kỹ thuật trong sản xuất lúa ở tỉnh Đồng Tháp. Nghiên cứu áp dụng phương pháp phân tích nhân tố cho thấy, mức độ hài lòng của nông hộ chịu tác động bởi các nhân tố: Sự cảm thông; Sự đảm bảo; Sự tin cậy; Phương tiện hữu hình.

Kết quả tổng quan tài liệu cho thấy đa phần các nghiên cứu sử dụng phương pháp phân tích nhân tố khám phá (EFA) và mô hình Logit thứ tự để đo lường mức độ hài lòng của nông hộ đối với chương trình khuyến nông. Nghiên cứu này sử dụng phương pháp nhân tố khẳng định (CFA) là bước tiếp theo của phương pháp phân tích nhân tố khám phá (EFA). Công cụ này được sử dụng để đánh giá tính hiệu lực của mô hình đo lường là độ phù hợp của mô hình với dữ liệu và tính hiệu lực của nhân tố (Hair \& ctv., 2010). Sau khi mô hình đo lường đã được đánh giá và đảm bảo tính hiệu lực, mô hình cấu trúc tuyến tính sẽ được ước lượng bằng SEM ở giai đoạn tiếp theo (Dang \& ctv., 2012).

\subsection{Nguồn số liệu}

Số liệu thứ cấp được thu thập từ Sở Nông nghiệp và Phát triển Nông thôn tỉnh Ninh Thuận, Trung tâm Khuyến nông tỉnh Ninh Thuận, Phòng Nông nghiệp huyện Ninh Phước.

Số liệu sơ cấp được thu thập bằng phương pháp chọn mẫu phân tầng kết hợp với ngẫu nhiên. Thông qua phỏng vấn trực tiếp 320 hộ đã tham gia các chương trình khuyến nông trên địa bàn 3 xã gồm xã Phước Hậu, xã Phước Hữu và xã An Hải tại huyện Ninh Phước bằng phiếu điều tra soạn sẵn.

\subsection{Phương pháp phân tích số liệu}

Mô hình cấu trúc tuyến tính (SEM) là một trong những kỹ thuật nghiên cứu được ứng dụng trong nhiều lĩnh vực nhờ tính linh hoạt trong việc mô hình hóa các quan hệ giữa các biến độc lập và phụ thuộc. Mục tiêu của ước lượng là đo lường mức độ hài lòng của nông hộ đối với chất lượng chương trình khuyến nông. Thang đo chất lượng dịch vụ thường được sử dụng nhất là thang đo 5 thành phần với 24 thuộc tính của Parasuraman \& ctv. (1988), bao gồm: (1) Phương tiện hữu hình (Tangible), (2) Mức độ đáp ứng (Responsiveness), (3) Độ tin cậy (Reliability), (4) Sự đảm bảo (Assurance), (5) Sự cảm thông (Empathy) (Hình 1).

Nhân tố phương tiện hữu hình được đo lường bằng 6 biến: Thiết bị giảng dạy đầy đủ (hh1); Thiết bị giảng dạy hiện đại (hh2); Nơi học tập thuận lợi (hh3); Nơi trình diễn các lớp học được 


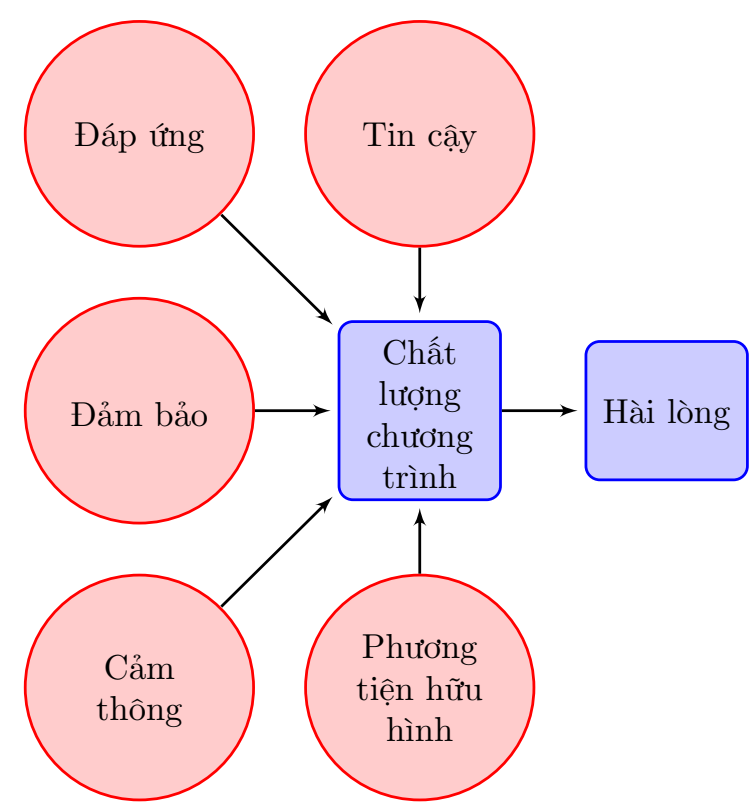

Hình 1. Mô hình đo lường mức độ hài lòng của nông hộ đối với chất lượng chương trình khuyến nông.

tổ chức chu đáo (hh4); Kết hợp tốt của ban tổ chức khuyến nông (hh5); Sự hỗ trợ của trung tâm trong các chương trình là phù hợp (hh6).

Nhân tố tin cậy được đo lường bằng 5 biến: Trung tâm khuyến nông luôn thực hiện đúng cam kết (tc1); Trung tâm khuyến nông luôn quan tâm sâu sắc trong các vấn đề nông dân gặp phải (tc2); Trung tâm khuyến nông cung cấp chính xác thông tin nông hộ cần (tc3); Trung tâm khuyến nông cung cấp thông tin đúng thời điểm (tc4); Trung tâm khuyến nông luôn thông báo thời gian thực hiện chương trình (tc5).

Nhân tố đáp ứng được đo lường bằng 4 biến: Cán bộ khuyến nông có kiến thức chuyên môn tốt (du1); Cán bộ khuyến nông luôn giúp đỡ nông hộ (du2); Cán bộ khuyến nông giải đáp thắc mắc thấu đáo (du3); Cán bộ khuyến nông tận tình hướng dẫn nông hộ trong thực hành (du4).

Nhân tố đảm bảo được đo lường bằng 5 biến: Nông hộ an tâm khi áp dụng các tiến bộ trong sản xuất (db1); Cách trình bày rõ ràng và hướng dẫn dễ hiểu (db2); Trao đổi trong lớp học sôi nổi, thoải mái (db3); Cán bộ khuyến nông có nhiều kinh nghiệm (db4); Hoạt động tham quan thực tế phù hợp (db5).

Nhân tố cảm thông được đo lường bằng 4 biến: Các hoạt động của chương trình khuyến nông phù hợp với nhu cầu nông hộ (ct1); Cán bộ khuyến nông thông cảm với những khó khăn của nông hộ (ct2); Hoạt động khuyến nông có thời gian làm việc thuận tiện (ct3); Cán bộ khuyến nông gần gũi và thân thiện (ct4).

Thang đo Likert (Likert, 1932) được sử dụng để đánh giá mức độ hài lòng: (5) Rất hài lòng; (4) Hài lòng; (3) Không ý kiến; (2) Không hài lòng; (1) Hoàn toàn không hài lòng.

Ước lượng hợp lý cực đại được thực hiện bởi phần mềm IBM SPSS Amos 20. Quá trình ước lượng gồm 2 giai đoạn: giai đoạn thứ nhất nhằm đánh giá hiệu lực của mô hình đo lường và giai đoạn thứ hai để kiểm định mô hình cấu trúc tuyến tính. Phân tích nhân tố khẳng định (CFA) được dùng trong giai đoạn thứ nhất. Hai tiêu chí cần thiết để mô hình đo lường có hiệu lực là mức chấp nhận về sự phù hợp của mô hình và hiệu lực của nhân tố (Hair \& ctv., 2010). Sự phù hợp của mô hình với dữ liệu có thể được đo bằng một trong số các chỉ tiêu như Chi-square điều chỉnh theo bậc tự do $\chi^{2} / d f$, chỉ số tích hợp so sánh (CFI); Chỉ số Tucker và Lewis (TLI) và chỉ số RMSEA. Đối với tính hiệu lực của nhân tố, ba chỉ tiêu quan trọng cần xem xét đó là hệ số tải nhân tố đã chuẩn hóa (standardized factor loadings), tổng phương sai trích trung bình (average variance extracted AVE) và độ tin cậy của nhân tố (construct reliability - CR).

Khi mô hình đo lường đã được kiểm định tính hiệu lực, ước lượng mô hình cấu trúc tuyến tính được thực hiện. Giai đoạn thứ hai là chạy mô hình SEM và sử dụng các chỉ tiêu đánh giá như với CFA. Sau đó là các diễn giải về hệ số đường dẫn, độ phù hợp với mô hình cấu trúc $\left(R^{2}\right)$, tác động trực tiếp, gián tiếp và tổng tác động.

\section{Kết Quả và Thảo Luận}

\subsection{Thực trạng hoạt động khuyến nông tại huyện Ninh Phước}

Một trong những kết luận giống nhau ở các cuộc điều tra kinh tế trong nông thôn là hiện nay nông dân Việt Nam kiến thức còn chưa cao. Từ đó sự ra đời của chính sách khuyến nông trở thành một yêu cầu bức xúc nhằm nâng cao sự hiểu biết của nông dân về những tiến bộ kỹ thuật mới trong sản xuất. Mục tiêu và nội dung chủ yếu của chính sách khuyến nông là truyền bá kiến thức cho nông dân ngay tại địa bàn sản xuất của họ, giúp họ đưa ra những quyết định đúng đắn trước những tình huống nảy sinh trong quá trình sản xuất mà 
không cần qua các lớp đào tạo tập trung ở trường học. Những kiến thức này được người nông dân tiếp cận bằng nhiều hình thức đa dạng.

Bảng 1. Hình thức khuyến nông tại địa phương

\begin{tabular}{lcc}
\hline $\begin{array}{l}\text { Các hình thức } \\
\text { khuyến nông }\end{array}$ & $\begin{array}{c}\text { Tần suất } \\
(\text { hộ })\end{array}$ & $\begin{array}{c}\text { Tỷ lệ } \\
(\%)\end{array}$ \\
\hline $\begin{array}{l}\text { Tập huấn từ cán bộ } \\
\text { khuyến nông }\end{array}$ & 304 & 44,25 \\
$\begin{array}{l}\text { Hội thảo tham quan } \\
\text { Mồ hình trình diễn }\end{array}$ & 192 & 27,95 \\
khuyến nông & 75 & 10,92 \\
Hội thảo chuyên đề & 116 & 16,88 \\
\hline
\end{tabular}

Kết quả nghiên cứu (Bảng 1) cho thấy, hình thức phổ biến nhất $(44,25 \%)$ là tổ chức các lớp tập huấn do các cán bộ trung tâm khuyến nông và trạm khuyến nông hướng dẫn trực tiếp. Ngoài ra, người nông dân còn tiếp cận các tiến bộ kỹ thuật qua các lớp hội thảo chuyên đề, hội thảo đầu bờ và mô hình trình diễn khuyến nông do các trạm khuyến nông huyện kết hợp với Hội Nông Dân địa phương.

Theo Wharton (1959) cho rằng với tất cả nguồn lực đầu vào giống nhau, hai nông dân với sự chênh lệch về trình độ kỹ thuật nông nghiệp sẽ có kết quả sản xuất khác nhau. Trình độ kiến thức nông nghiệp có tác động cùng chiều đến thu nhập của nông dân ở Việt Nam (Dinh, 2008). Kiến thức nông nghiệp có thể được xem xét bởi mức độ tham gia của nông dân vào các hoạt động khuyến nông. Kết quả khảo sát tại vùng nghiên cứu (Bảng 2) cho thấy, mức độ tham gia của nông hộ vào chương trình khuyến nông trung bình 2 lần trong năm chiếm tỷ lệ $47,50 \%$ và 3 lần trong năm chiếm tỷ lệ 20,00\% . Điều này cho thấy một sự tích cực nâng cao những kiến thức mới từ người nông dân và thành quả của chương trình khuyến nông.

Bảng 2. Số lần tham gia khuyến nông của nông hộ

\begin{tabular}{ccc}
\hline Số lần tham gia & Tần số (hộ) & Tỉ lệ $(\%)$ \\
\hline 1 lần & 71 & 22,19 \\
2 lần & 152 & 47,50 \\
3 lần & 64 & 20,00 \\
Trên 4 lần & 33 & 10,31 \\
\hline
\end{tabular}

Trong số các nông hộ tham gia khuyến nông thì lĩnh vực tham gia khuyến nông chủ yếu tại huyện là trồng trọt chiếm tỷ lệ $70,66 \%$; nội dung của các buổi tham gia này nhằm định hướng cho nông dân sản xuất theo nhu cầu nâng cao chất lượng và thu nhập dựa trên cơ sở ứng dụng hợp lý những tiến bộ kỹ thuật bao gồm biện pháp cải tạo đất, sử dụng giống mới, sử dụng phân bón và thuốc bảo vệ thực vật, các biện pháp bảo quản sau thu hoạch.

\section{2. Đo lường mức độ hài lòng của nông hộ đối với chất lượng dịch vụ chương trình khuyến nông}

\subsection{1. Đánh giá độ tin cậy thang đo với hệ số Cron- bach's Alpha}

Chất lượng chương trình khuyến nông được đo lường bởi 5 nhân tố là Hữu hình gồm 6 chỉ tiêu, Tin cậy gồm 5 chỉ tiêu, Đáp ứng gồm 4 chỉ tiêu, Đảm bảo gồm 5 chỉ tiêu và Cảm thông gồm 4 chỉ tiêu. Tổng cộng có 24 biến quan sát được sử dụng.

Kết quả kiểm định độ tin cậy thang đo với hệ số Cronbach's Alpha thể hiện trong Bảng 3 cho thấy các nhân tố của chất lượng dịch vụ đều có hệ số Cronbach's Alpha được chấp nhận đó là lớn hơn 0,6 (Nunnally, 1978; Peterson, 1994). Hệ số tương quan biến tổng của các biến trong thang đo đều lớn hơn 0,3 nên đạt yêu cầu (Hair \& ctv., 2010), do đó các biến đo lường của các nhân tố này đều được sử dụng cho phân tích EFA.

\subsubsection{Phân tích nhân tố khám phá EFA}

Kết quả EFA với phép trích nhân tố được sử dụng là Principal Axis Factoring và phép quay không vuông góc Promax cho thấy, có 5 nhân tố được rút trích ra với phương sai trích là $69,71 \%$ $(>50 \%)$ dạt yêu cầu. KMO là $0,757(>0,5)$ và kiểm định Bartlett có ý nghĩa thống kê ( $\mathrm{Sig}<$ $0,05)$ nên phân tích EFA là phù hợp. Tuy nhiên, trong 24 biến quan sát có 6 biến quan sát (hh5, hh6, tc4, tc5, du3, du4) có hệ số tải nhân tố (Factor loading) nhỏ hơn 0,5 lần lượt bị loại ra khỏi mô hình (Gerbing \& Anderson, 1988). Sau khi loại 6 biến không đạt yêu cầu, kết quả EFA lần cuối được trình bày trong Bảng 4 .

\subsubsection{Kết quả kiểm định thang đo bằng CFA}

Kết quả CFA (Hình 2) cho thấy trọng số các biến quan sát đều đạt chuẩn cho phép $(\geq 0,5)$ và có ý nghĩa thống kê (các giá trị $\mathrm{p}$ đều bằng 0,000 ); thấp nhất là trọng số yếu tố đáp ứng 0,60 . Như vậy có thể kết luận các biến quan sát dùng để đo lường 5 nhân tố của thang đo chất lượng dịch vụ 
Bảng 3. Cronbach's Alpha các nhân tố thang đo chất lượng dịch vụ

\begin{tabular}{ccc}
\hline Thang đo & Biến đặc trưng & Cronbach's Alpha \\
\hline Phương tiện hữu hình & HH1, HH2, HH3, HH4, HH5, HH6 & 0,897 \\
Tin cậy & TC1, TC2, TC3, TC4, TC5 & 0,801 \\
Đáp ứng & DU1, DU2, DU3, DU4 & 0,655 \\
Đảm bảo & ĐB1, ĐB2, ĐB3, ĐB4, ĐB5 & 0,843 \\
Cảm thông & CT1, CT2, CT3, CT4 & 0,825 \\
Chất lượng & CL1, CL2, CL3 & 0,766 \\
Hài lòng & HL1, HL2, HL3 & 0,830 \\
\hline
\end{tabular}

Bảng 4. Kết quả EFA của thang đo chất lượng dịch vụ

\begin{tabular}{|c|c|c|c|c|c|c|}
\hline \multirow{2}{*}{\multicolumn{2}{|c|}{ Biến quan sát }} & \multicolumn{5}{|c|}{ Nhân tố } \\
\hline & & 1 & 2 & 3 & 4 & 5 \\
\hline ĐB4 & Cán bộ khuyến nông có nhiều kinh nghiệm & 0,820 & & & & \\
\hline ĐB3 & Trao đổi trong lớp học sôi nổi, thoải mái & 0,798 & & & & \\
\hline ĐB2 & Cách trình bày rõ ràng và hướng dẫn dễ hiểu & 0,779 & & & & \\
\hline ĐB1 & $\begin{array}{l}\text { Nông hộ an tâm khi áp dụng các tiến bộ trong } \\
\text { sản xuất }\end{array}$ & 0,685 & & & & \\
\hline ĐB5 & Hoạt động tham quan thực tế phù hợp & 0,642 & & & & \\
\hline HH3 & Nơi học tập thuận lợi & & 0,911 & & & \\
\hline $\mathrm{HH} 4$ & Nơi trình diễn các lớp học được tổ chức chu đáo & & 0,910 & & & \\
\hline HH1 & Thiết bị giảng dạy đầy đủ & & 0,871 & & & \\
\hline $\mathrm{HH} 2$ & Thiết bị giảng dạy hiện đại & & 0,797 & & & \\
\hline CT1 & $\begin{array}{l}\text { Các hoạt động của chương trình khuyến nông } \\
\text { phù hợp với nhu cầu nông hộ }\end{array}$ & & & 0,871 & & \\
\hline CT3 & $\begin{array}{l}\text { Hoạt động khuyến nông có thời gian làm việc } \\
\text { thuận tiện }\end{array}$ & & & 0,806 & & \\
\hline $\mathrm{CT} 2$ & $\begin{array}{l}\text { Cán bộ khuyến nông thông cảm với những } \\
\text { khó khăn của nông hộ }\end{array}$ & & & 0,790 & & \\
\hline $\mathrm{CT} 4$ & Cán bộ khuyến nông gần gũi và thân thiện & & & 0,776 & & \\
\hline TC2 & $\begin{array}{l}\text { Trung tâm khuyến nông luôn quan tâm sâu sắc } \\
\text { trong các vấn đề nông dân gặp phải }\end{array}$ & & & & 0,808 & \\
\hline TC1 & $\begin{array}{l}\text { Trung tâm khuyến nông luôn thực hiện đúng } \\
\text { cam kết }\end{array}$ & & & & 0,804 & \\
\hline TC3 & $\begin{array}{l}\text { Trung tâm khuyến nông cung cấp chính xác } \\
\text { thông tin nông hộ cần }\end{array}$ & & & & 0,735 & \\
\hline DU2 & Cán bộ khuyến nông luôn giúp đỡ nông hộ & & & & & 0,864 \\
\hline DU1 & Cán bộ khuyến nông có kiến thức chuyên môn tốt & & & & & 0,838 \\
\hline
\end{tabular}

đều đạt giá trị hội tụ.

Kết quả cũng cho thấy mô hình có 124 bậc tự do, giá trị kiểm đinh Chi-square $=201,981$ với $P_{\text {value }}=0,000 ;$ Chi-square $/ \mathrm{df}=1,629 \leq 2$ và các chỉ số TLI $=0,969 ; \mathrm{CFI}=0,978 \geq 0,9 ; \mathrm{RMSEA}$ $=0,044 \leq 0,08$ thì mô hình phù hợp với dữ liệu nghiên cứu (Nguyen, 2008). Mức độ phù hợp của mô hình với dữ liệu nghiên cứu cho thấy điều kiện cần và đủ cho tập biến quan sát đạt tính đơn hướng, trừ trường hợp các nhân tố của các biến quan sát có tương quan nhau (Steenkamp
\& Van Trijp, 1991) vì vậy các nhân tố Đảm bảo, Cảm thông, Tin cậy, Đáp ứng đạt được tính đơn hướng.

Sau khi kiểm định tính hiệu lực của mô hình đo lường từ kết quả phân tích CFA, phân tích SEM được thực hiện với các chỉ tiêu đo lường độ phù hợp của mô hình với dũ liệu tương tự CFA như sau : Chi-square $=409,552$ với $\mathrm{p}_{\text {value }}=0,000$; Chi-square $/ \mathrm{df}=1,735 \leq 2$ và các chỉ số $\mathrm{TLI}=$ $0,953, \mathrm{CFI}=0,963 \geq 0,9, \mathrm{RMSEA}=0,048 \leq$ 0,08. Kết quả mô hình cấu trúc tuyến tính được 


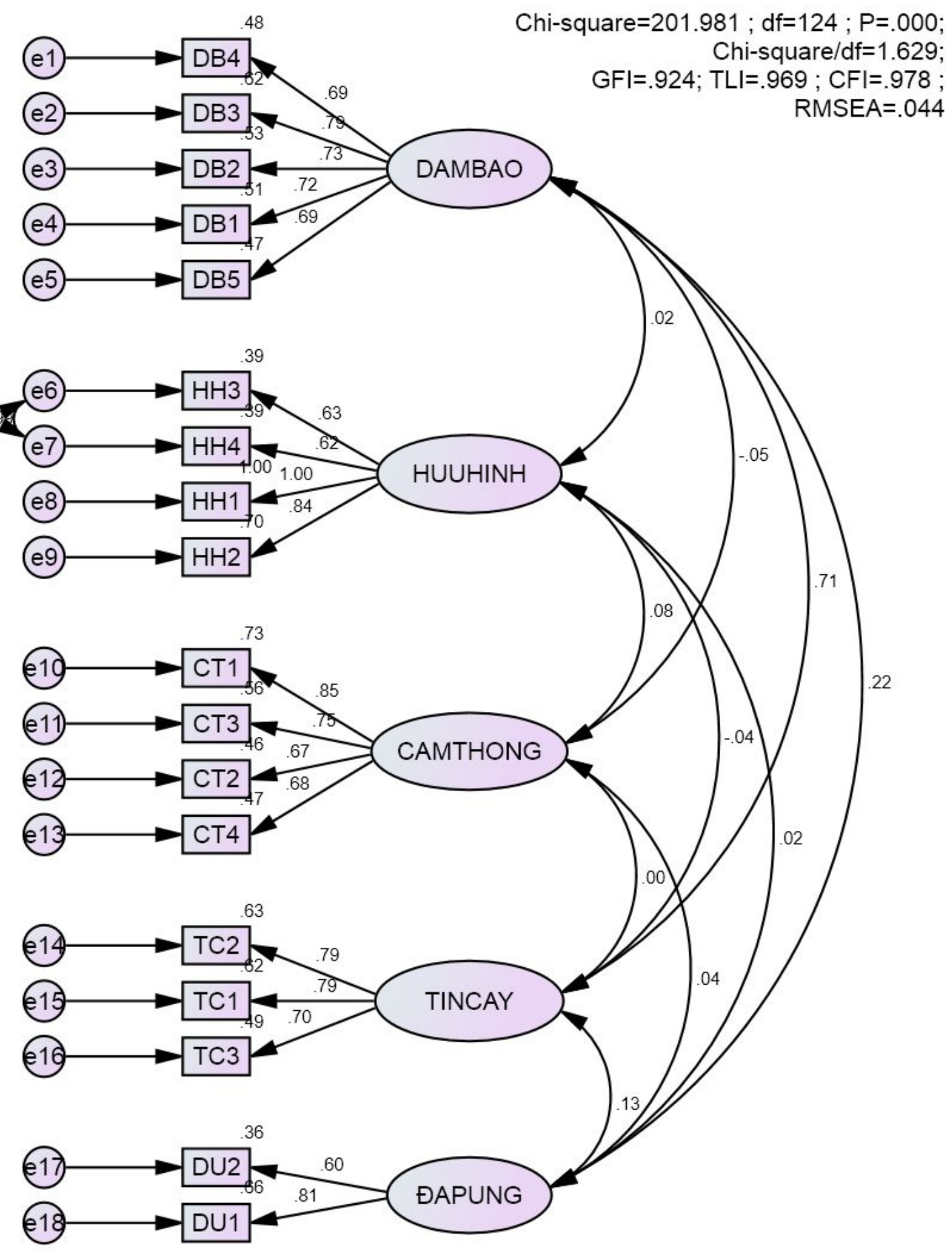

Hình 2. Kết quả CFA.

thể hiện ở Hình 3 và Bảng 5 . 


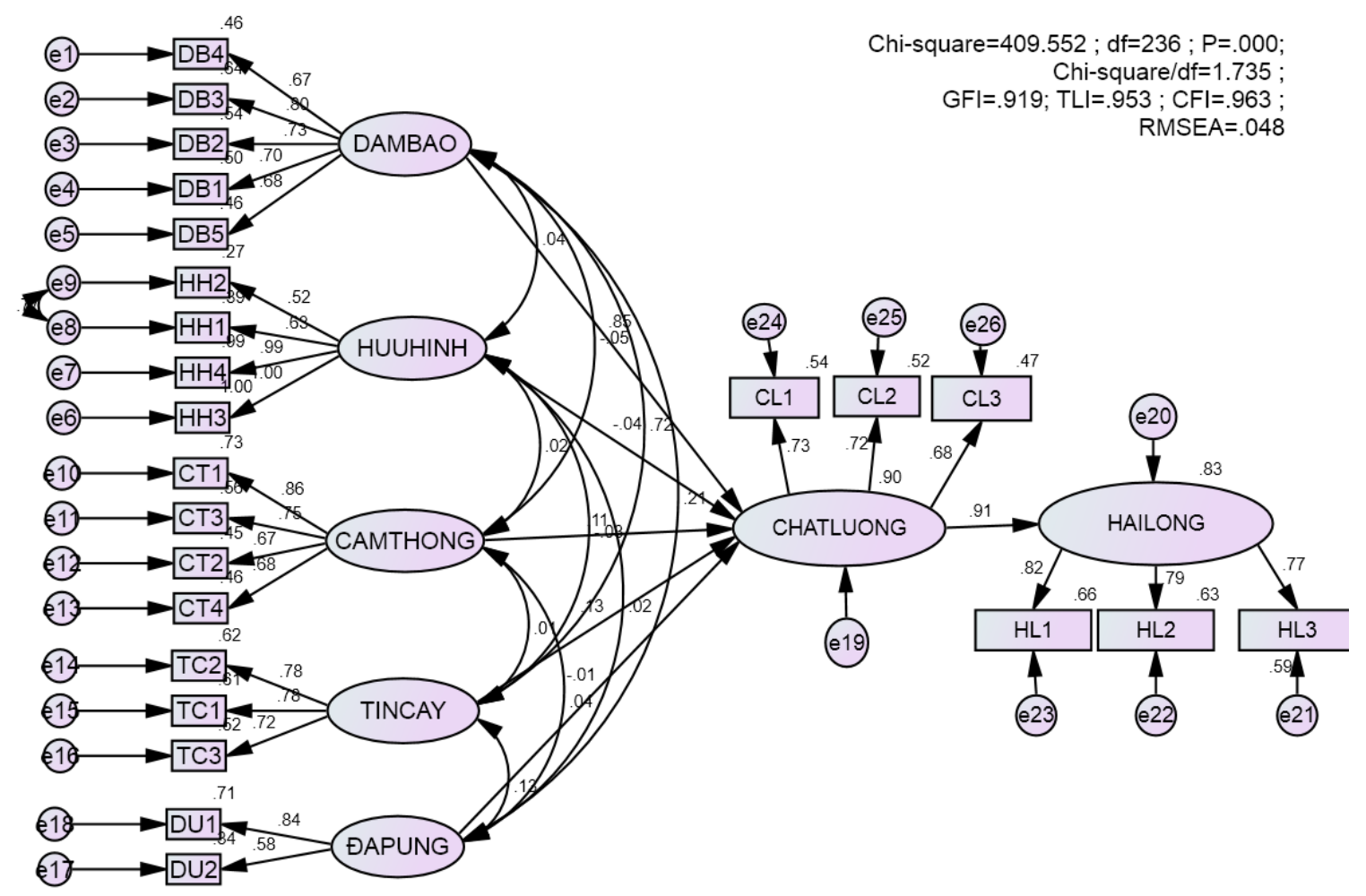

Hình 3. Kết quả SEM.

Bảng 5. Kết quả mô hình SEM - Các tham số ước lượng

\begin{tabular}{lcccc}
\hline Mối quan hệ & Ước lượng & $\mathrm{SE}$ & $\mathrm{CR}$ & $\mathrm{P}_{\text {value }}$ \\
\hline Chất lượng $\leftarrow$ Hữu hình &,- 075 &, 078 &,- 961 &, $337^{n s}$ \\
Chất lượng $\leftarrow$ Đảm bảo &, 668 &, 055 & 12,136 &, $000^{* * *}$ \\
Chất lượng $\leftarrow$ Cảm thông &, 082 &, 029 & 2,842 &, $004^{* * *}$ \\
Chất lượng $\leftarrow$ Tin cậy &, 119 &, 053 & 2,239 &, $025^{* *}$ \\
Chất lượng $\leftarrow$ Đáp ứng &,- 019 &, 064 &,- 293 &, $770^{n s}$ \\
Hài lòng $\leftarrow$ Chất lượng &, 982 &, 071 & 13,851 &, $000^{* * *}$ \\
\hline *** Có ýn
\end{tabular}
có ý nghĩa thống kê $(P>0,05)$.

Theo Flores \& Sarandón (2004), sự hài lòng của người nông dân về chương trình khuyến nông là yếu tố quan trọng của sự bền vững và sự bền vững trở thành mục tiêu hàng đầu của các nhà nghiên cứu khoa học và nhà làm chính sách. Kết quả nghiên cứu ở Bảng 5 cho thấy, chất lượng dịch vụ chương trình khuyến nông có ảnh hưởng thuận chiều và tương quan mạnh tới sự hài lòng của nông hộ thể hiện qua hệ số $\beta=0,982$ và ước lượng này đạt ý nghĩa thống kê tại $P=0,000$. Điều này có nghĩa là sự hài lòng của người nông dân tăng lên khi chất lượng dịch vụ chương trình khuyến nông tại địa phương được nâng cao. Cụ thể, kết quả mô hình SEM chỉ ra rằng nếu trung tâm khuyến nông luôn thực hiện đúng cam kết, luôn quan tâm sâu sắc trong các vấn đề nông dân gặp phải và cung cấp chính xác thông tin nông hộ cần thì chất lượng dịch vụ chương trình khuyến nông sẽ tăng lên rõ rệt. Nếu nông hộ an tâm khi áp dụng các tiến bộ trong sản xuất, cách trình bày rõ ràng và hướng dẫn dễ hiểu của cán bộ khuyến nông, trao đổi trong lớp học sôi nổi, thoải mái, cán bộ khuyến nông có nhiều kinh nghiệm và các hoạt động tham quan thực tế phù hợp sẽ làm tăng chất lượng dịch vụ chương trình 
khuyến nông tại địa phương. Nếu các hoạt động của chương trình khuyến nông phù hợp với nhu cầu nông hộ, cán bộ khuyến nông thông cảm với những khó khăn của nông hộ, hoạt động khuyến nông có thời gian làm việc thuận tiện và cán bộ khuyến nông gần gũi, thân thiện với người nông dân thì sẽ làm chất lượng dịch vụ chương trình khuyến nông của địa phương cải thiện.

Trong ba nhân tố được nghiên cứu có tác động đến chất lượng dịch vụ chương trình khuyến nông thì nhân tố đảm bảo có ảnh hưởng lớn nhất. Như vậy có thể nói để nâng cao chất lượng dịch vụ chương trình khuyến nông thì các chương trình khuyến nông tại địa phương phải làm sao cho nông hộ an tâm khi áp dụng các tiến bộ trong sản xuất, trao đổi trong lớp học sôi nổi thoải mái, cán bộ khuyến nông có nhiều kinh nghiệm, trình bày rõ ràng dễ hiểu và các hoạt động tham quan thực tế phải phù hợp.

\section{Kết Luận}

Kết quả phân tích mô hình cấu trúc tuyến tính (SEM) cho thấy sự phù hợp của mô hình lý thuyết với chất lượng dịch vụ chương trình khuyến nông cũng như sự hài lòng của nông hộ đối với hoạt động khuyến nông tại địa phương. Nhóm nhân tố đảm bảo có ảnh hưởng lớn nhất đến sự hài lòng của nông hộ đến chất lượng dịch vụ chương trình khuyến nông. Các nhóm nhân tố khác như độ tin cậy của chương trình khuyến nông và sự cảm thông của cán bộ khuyến nông cũng có ảnh hưởng quan trọng đến sự hài lòng của nông hộ đối với chất lượng dịch vụ chương trình khuyến nông. Từ những kết quả nghiên cứu này, để nâng cao chất lượng dịch vụ chương trình khuyến nông tại huyện Ninh Phước thì địa phương cần quan tâm đến việc đảm bảo về cơ sở vật chất phục vụ đào tạo và tổ chức hoạt động khuyến nông. Việc đảm bảo cơ sở vật chất nhằm hướng dẫn, giúp đỡ nông hộ dễ tiếp cận cũng như ứng dụng các tiến bộ kỹ thuật vào sản xuất nông nghiệp. Ngoài ra, đội ngũ cán bộ khuyến nông là một trong những đội ngũ nòng cốt cần tiếp tục nâng cao trình độ chuyên môn thông qua các lớp tập huấn ngắn hạn về những thay đổi của tiến bộ khoa học trong sản xuất nông nghiệp để truyền đạt hướng dẫn người nông dân tiếp thu và ứng dụng khoa học kỹ thuật một cách đầy đủ và chính xác.

\section{Tài Liệu Tham Khảo (References)}

Cronin, J. J., \& Taylor, S. A. (1992). Measuring Service Quality: A Reexamination and Extension. Journal of Marketing 56(3), 55-68.

Dang, L. H., Li, E., \& Bruwer (2012). Understanding climate change adaptive behaviour of farmers: An integrated conceptual framework. The International Journal of Climate Change: Impacts \& Responses 3(2), 255-272.

Dinh, H. P. (2008). Agricultural extension, "the gold key" for the integration of farmers. Communist Review 15. Retrieved March 21, 2008, from http://www.tapchicongsan.org.vn/Khuyen-nong-chiakhoa-vang-cua-nong-dan.aspx.

Elias, A., Nohmi, M., \& Ishida, A. (2015). Farmer's satisfaction with agricultural extension service and its influencing factors in North West Ethiopia. Journal Agricultural Science and Technology 18(1), 39-53.

Flores, C. C., \& Sarandón, S. J. (2004). Limitations of neoclassical economics for evaluating sustainability of agricultural systems: Comparing organic and conventional systems. Journal of Sustainable Agriculture $24(2), 77-91$

Gerbing, D. W., \& Anderson, J. C. (1988). Structural Equation Modeling in Practice: A Review and Recommended Two-Step Approach. Psychological Bulletin 103(3), 411-423.

Hair, J. F. J., Black, W. C., Babin, B. J., \& Anderson, R. E. (2010). Multivariate data analysis $\left(7^{\text {th }}\right.$ ed.). Cambridge, United Kingdom: Pearson.

Kotler, P., \& Keller, K. L. (2006). Marketing Management. New York, USA: Pearson Prentice Hall.

Likert, R. A. (1932). A technique for the measurement of attitudes. Archives of Psychology 22(140), 5-53.

Lin (2003). A critical appraisal of customer satisfaction and ecommerce. Managerial Auditing Journal 18(3), 20

Nguyen, N. Q., Le, H. T. D., Hoang, L. T. H., \& Tran, Y. L. H. (2011). Factors influencing household satisfaction with training methods on technological application on rice cultivation in Dong Thap province. Can Tho University Journal of Science 17b, 97-105.

Nguyen, T. D. (2008). Methods for scientific research in bussiness. Ha Noi, Vietnam: Labor and Social Publisher.

Nguyen, T. H., \& Nguyen, C. N. (2016). Agricultural and fishery extension policies in some countries and lessons learnt for aquacultural extension policies in coastal zones in the north of Vietnam. Vietnam Journal of Agricultural Sciences 14(2), 202-210.

Nunnally, J. (1978). Psychometric Theory. New York, USA: McGraw Hill. 
Olajide, P. O. (2011). Causual Direction Between Satisfaction and Service Quality: A Review of Literature. Europe Journal of Humanities and Social Science 2(1), 88-96.

Parasuraman, A. P., Zeithaml, A. A., \& Berry, L. (1988). Servqual: A Multi - Item Scale for Measuring Consumer Perception of Service Quality. Journal of Retailing 64(1), 21-40.

Peterson (1994). A Meta-Analysis of Cronbach's Cofficient Alpha. Journal of Consumer Research 21(2), 3891.

Pham, N. N., Huynh, T. Q., \& Do, P. N. D. (2014). Determining the factors influencing the farmers' satisfaction via the FFS training course about strengthening farmer capacity on rice breeding and seed production in Hau Giang Province in 2012. Can Tho University Journal of Science 34c, 62-73.
Steenkamp, J. B. E. M., \& Van Trijp, H. C. M. (1991). The Use of LISREL in Validating Marketing Constructs. International Journal of Research in Marketing 8(4), 283-299.

Wayne, G. G., Nicole, W., \& Lendel, K. (2014). Farmer's satisfaction with extension services in the Organization of Eastern Caribbean States. Journal of International Agricultural and Extension Education 21(3), 49-62.

Wharton, C. A. (1959). The U.S Graduate Training of Asian Agricultural Economists. NewYork, USA: Council on Economic and Cultural Affairs. 